\title{
Experimental study on onset turbulence in a flat-plate boundary layer
}

\author{
Kensuke OKADA*, Masato HIROTA*, Seiichiro IZAWA* and Yu FUKUNISHI* \\ * Department of Mechanical Systems Engineering, Tohoku University \\ 6-6-01 Aramaki-Aoba, Aoba-ku, Sendai 980-8579, Japan \\ E-mail: izawa@fluid.mech.tohoku.ac.jp
}

Received: 11 June 2019; Revised: 4 August 2019; Accepted: 21 August 2019

\begin{abstract}
A wind tunnel experiment was performed to investigate the suitable conditions in which a localized turbulent region can easily be generated in a flat-plate boundary layer. An artificial disturbance of zero-mass flux was introduced upstream using a combination of a short-duration jet and suctions to prepare a potentially unstable environment. The disturbed region by itself decayed downstream. Another jet was then ejected downstream at several different timings and two different spanwise locations relative to the passage of the locally disturbed region to promote transition to turbulence. Although the jet was too weak to trigger the turbulence transition by itself, an isolated turbulent region, the so-called turbulent spot, was generated when ejected against the disturbed region. The optimum conditions were found when the jet was ejected between the high- and low-speed areas of the convecting unstable region.
\end{abstract}

Keywords : Boundary layer, Local unstable region, Short-duration jet, Onset of turbulence, Turbulent spot

\section{Introduction}

The transition to turbulence in boundary layers follows different paths depending on the freestream turbulence level. In a low-disturbance environment, the transition occurs by way of the exponential growth of the Tollmien-Schlichting (TS) waves. This is a slow process because the TS waves are generated as a consequence of viscous instability whose growth rate is much smaller than that of inflection point instability, and it then takes a long time before inducing the secondary instability (Herbert 1988). The transition occurs early when the freestream turbulence level exceeds $1 \%$, thereby bypassing the TS wave formation. Streaky structures, which are elongated in the streamwise direction and aligned in the spanwise direction, are created in the boundary layer and undergo an algebraic growth. These streaky structures generally have inflection points in both the wall-normal and spanwise directions, which cause them to break down in varicose or sinuous mode (Eloffson et al. 1999; Asai et al. 2007; Schlatter et al. 2008). Localized turbulent regions known as "turbulent spots" are randomly formed at the late stage of the transition process. These spots grow both in the streamwise and spanwise directions and eventually merge, resulting in a fully developed turbulent boundary layer (Narashima 1985). Therefore, revealing what kind of flow structures or conditions leads to the generation of turbulent spots at the onset of the turbulence is important.

Turbulent spots have been widely studied by many researchers after the first experimental observation by Emmons (1951), and Schubauer and Klebanoff (1955). Through a flow visualization experiment, Perry et al. (1981) suggested that turbulent spots consist of an array of $\Lambda$-shaped vortices. Sankaran et al. (1991) and Seifert et al. (1994) confirmed the existence of many $\Lambda$-shaped vortices inside turbulent spots using a rake of hotwire probes. Makita and Nishizawa (2001) pointed out that strong $\Lambda$-shaped vortices are created where two neighboring spots merge, maintaining their geometric identity further downstream even inside the turbulent boundary layer turning into turbulent bulges. The characteristics of turbulent spots under various conditions have also been investigated. Zhong et al. (2000) experimentally investigated the growth of turbulent spots under an adverse pressure gradient and reported that the spreading angle of turbulent spots 
became larger under a large adverse pressure gradient. Fransson et al. (2010) investigated the characteristics of turbulent spots under the same condition as the simulation done by Levin et al. (2007), where the boundary layer thickness was kept constant through a suction at the wall (asymptotic suction boundary layer). They showed that the traveling speed and the spread angle of turbulent spots depend on their generation conditions and on the Reynolds number.

An electrical spark or a "strong" short-duration jet from a hole in the surface is often used to artificially generate a turbulent spot in a laminar boundary layer. However, in a natural flow field, the turbulent spots appear one after another even in the absence of such a large stimulation. In other words, a turbulent spot should be generated using a much weaker stimulus if an appropriate flow condition is prepared. Streaky structures are a possible candidate building block for a locally unstable environment and suitable for turbulent spots because they always appear near the wall in the bypass transition process. The critical amplitude of the streak strength of the sinuous instability mode is approximately $26 \%$ of the freestream velocity, and that of the varicose instability mode is $37 \%$ (Andersson et al. 2001). The former sinuous mode associated with the wake-type profile across the low-speed streak can exponentially grow further downstream. The latter varicose mode is more stable because it is essentially governed by the Kelvin-Helmholtz instability of the inflectional velocity profile in the wall-normal direction. It can occur when the width of the low-speed streak is larger than the shear layer thickness (Asai et al. 2002). Uehara et al. (2011) investigated the suitable condition for the easy generation of turbulent spots by changing the location of the jet ejection relative to the streaky structures. They showed that turbulent spots could be easily generated when the jet is ejected into a low-speed region of the streaky structure. Yoshikawa et al. (2018) performed a direct numerical simulation to determine a key structure for the transition of a flat-pate boundary layer with streaky structures. They introduced a short-duration jet against the low-speed streak and found that the formation of an inclined streamwise vortex was key to the transition of the boundary layer.

The present study performed a wind tunnel experiment to investigate the suitable conditions in which the localized turbulent region can be easily excited in the Blasius boundary layer by a weak stimulus. A locally unstable environment was prepared upstream by a combination of a short-duration jet and suctions. Another jet was then ejected downstream to trigger the generation of a turbulent spot. The timing and location of the downstream jet was changed.

\section{Experimental setup}

The experiments were conducted in a blowout-type wind tunnel with a rectangular test section of $2000 \mathrm{~mm}$ in length and $500 \mathrm{~mm} \times 500 \mathrm{~mm}$ in cross-section. The wind tunnel had a settling chamber of $1500 \mathrm{~mm} \times 1500 \mathrm{~mm}$. The ratio of contraction from the settling chamber to the test section was 9:1. The wind tunnel ceiling was adjustable in the vertical direction such that the pressure gradient in the streamwise direction can be regulated. Figure 1 shows a schematic view of the experimental setup. A flat plate made of aluminum alloy was horizontally mounted $185 \mathrm{~mm}$ away from the bottom floor of the test section to avoid the contamination caused by the boundary layer, which developed along the inner wall of the wind tunnel. The plate measured $1610 \mathrm{~mm}$ long, $490 \mathrm{~mm}$ wide, and $10 \mathrm{~mm}$ thick. An elliptical sharp leading edge with a radius ratio of 24:1 was used to suppress the receptivity to the freestream turbulence. A flap with a chord length of $190 \mathrm{~mm}$ was used to adjust the stagnation point at the leading edge to prevent separation at the upper surface of the leading edge. The origin of the coordinate system was located at the center of the leading edge of the plate, where the $x, y$, and $z$ axes represent the streamwise, wall-normal, and spanwise directions, respectively. The freestream velocity $U_{\infty}$ was fixed at $8.5 \mathrm{~m} / \mathrm{s}$ all throughout the experiments. The ratio of the velocity fluctuation intensity to the freestream velocity in the test section was less than $0.3 \%$ of $U_{\infty}$. The variations of the pressure coefficients $C_{p}$ in the test section were adjusted to be less than $1 \%$; hence, the pressure gradient in the streamwise direction was negligibly small.

Three small holes with a diameter of $1 \mathrm{~mm}$ were opened in the plate surface at $x=300 \mathrm{~mm}$ as the Local Flow Destabilizer. Here, the center hole was placed at $z=0 \mathrm{~mm}$, and the other two holes were positioned on both sides 10 $\mathrm{mm}$ apart in the spanwise direction from the center hole. A circular part of the plate around the Local Flow Destabilizer was designed to be replaceable such that its configuration can be easily changed. One more hole was prepared $250 \mathrm{~mm}$ downstream from the three holes at $x=550 \mathrm{~mm}$ and $z=0 \mathrm{~mm}$, which was used to eject a jet that triggered the turbulent spot generation (Triggering Jet). Each hole was connected to a loudspeaker (VISATON, FRS7) beneath the plate via a funnel and a $6 \mathrm{~mm}$-diameter tube. The driving signal of the loudspeakers was provided by a personal computer via a digital-analog (D/A) converter (INTERFACE, PCI-3300) and a power amplifier. The signal was intended to push the fluid out in the funnel in a short time and slowly suck in the fluid afterward or the opposite. The function is given as follows:

$$
\operatorname{Amp}(t)=\left\{\begin{array}{lc}
-\frac{A}{2}+A t & (0 \leq t<T) \\
-\frac{A}{2}+A \exp \{-0.0005(t-T)\} & (T \leq t),
\end{array}\right.
$$




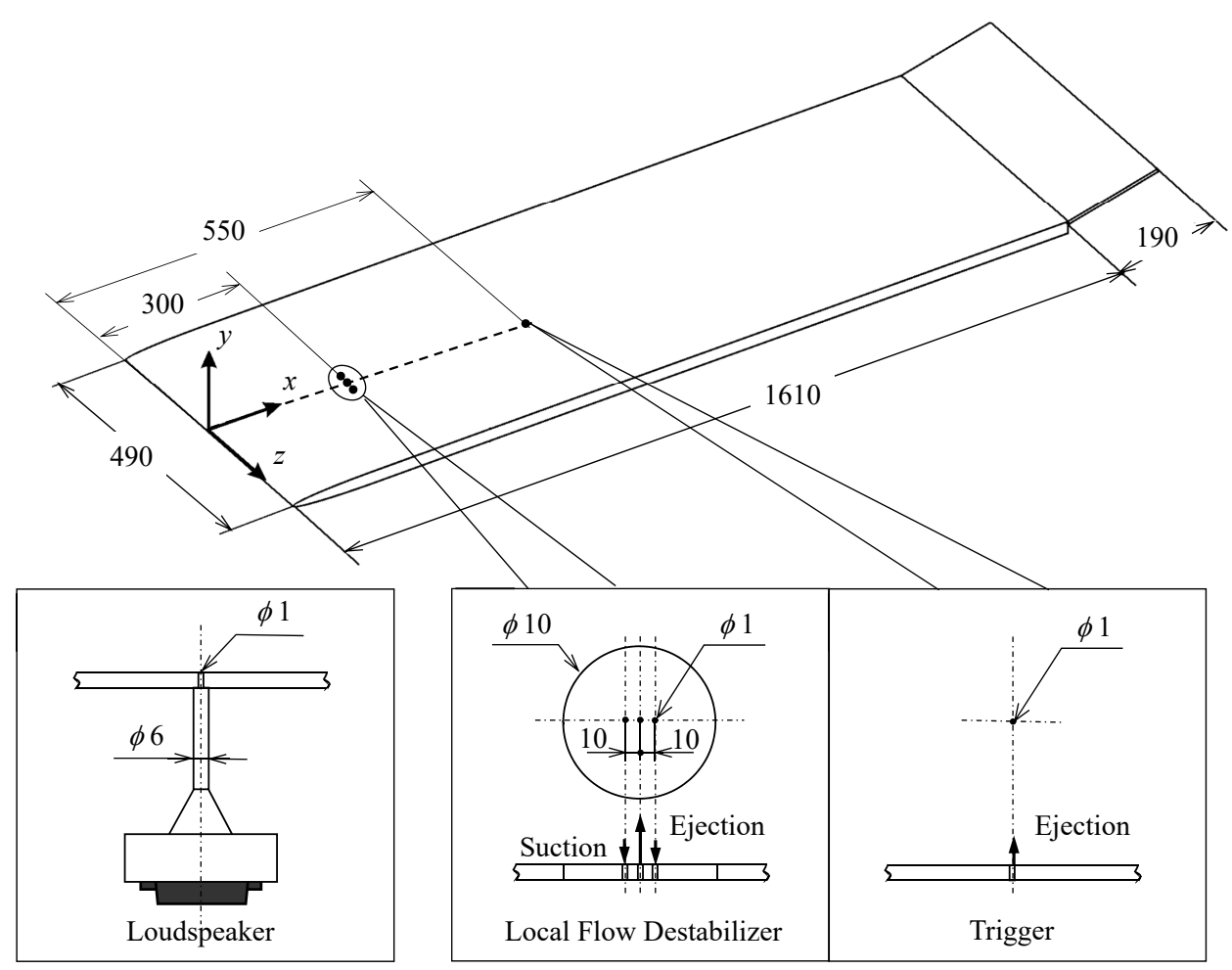

Fig. 1 Schematic view of the experimental setup.

where $A$ is the maximum voltage and $T$ is the rise time of the driving signal. A short-duration jet was issued from the small hole by operating the loudspeakers with this signal. The strength of the jet ejection $v_{\text {jet }}$ could be controlled by changing the input signal amplitude. In the present experiment, $v_{\text {jet }}$ was defined as the maximum value of the ensemble-averaged velocity measured at $1 \mathrm{~mm}$ above the ejection hole. For the Local Flow Destabilizer, $v_{\text {jet }}$ was set to $1.7 \mathrm{~m} / \mathrm{s}$, which was $20 \%$ of the freestream velocity $U_{\infty}$. For the Triggering Jet downstream, $v_{\text {jet }}$ was $1.5 \mathrm{~m} / \mathrm{s}$, which was $18 \%$ of $U_{\infty}$. A signal whose sign is opposite to the ejection case was used to apply suction. Measuring the strength of the suction using a hotwire probe was difficult; thus, the velocity produced by the suction was assumed to be the same as that of the jet case. The intensities of the suctions from the two holes of the Local Flow Destabilizer $v_{\text {suc }} / U_{\infty}$ were both set as half of the central jet 0.10 such that the net mass flow would be zero.

A single-type hotwire probe attached to a three-dimensional (3D) traversing mechanism was used with the constanttemperature anemometry (CTA) circuit to measure the velocity. The spatial resolution of 3D traversing mechanism was $0.2 \mathrm{~mm}$ in the $x$ direction, $0.01 \mathrm{~mm}$ in the $y$ direction, and $0.25 \mathrm{~mm}$ in the $z$ direction. A fourth-order Butterworth-type low-pass filter with a cut-off frequency of $2.5 \mathrm{kHz}$ was used to filter out the high-frequency components of the signals coming from the hotwire anemometer to prevent aliasing errors. The filtered signal was converted to digital data and stored in a personal computer via a 16 bit analog-digital (A/D) converter at a sampling frequency of $5 \mathrm{kHz}$. The number of data for one series sampling was $2^{17} \sim 2^{19}$.

The measurement data were processed by assuming that the Taylor's frozen-eddy hypothesis was applicable. This hypothesis is valid when the traveling velocity of a vortex is much larger than its deformation velocity. It is generally valid in boundary layer flows because the spatial structures associated with turbulence move downstream only slowly changing their shapes. Therefore, the time series data measured by the hotwire probe can be converted to spatial data. The relation between the time series data and the spatial data is presented as follows:

$$
x=-\bar{u}_{b} t
$$

where $\bar{u}_{b}$ is the local time-averaged velocity of the background flow field. Here, the time $t$ is an elapsed time after the Local Flow Destabilizer is activated.

There are several definitions of the turbulence intensity. Among them, the rms values of the velocity fluctuations from the time-averaged value are widely employed. However, using the values in flows with periodically fluctuating velocities is not appropriate because the periodic and ordered velocity fluctuations will be included in the measured turbulence 


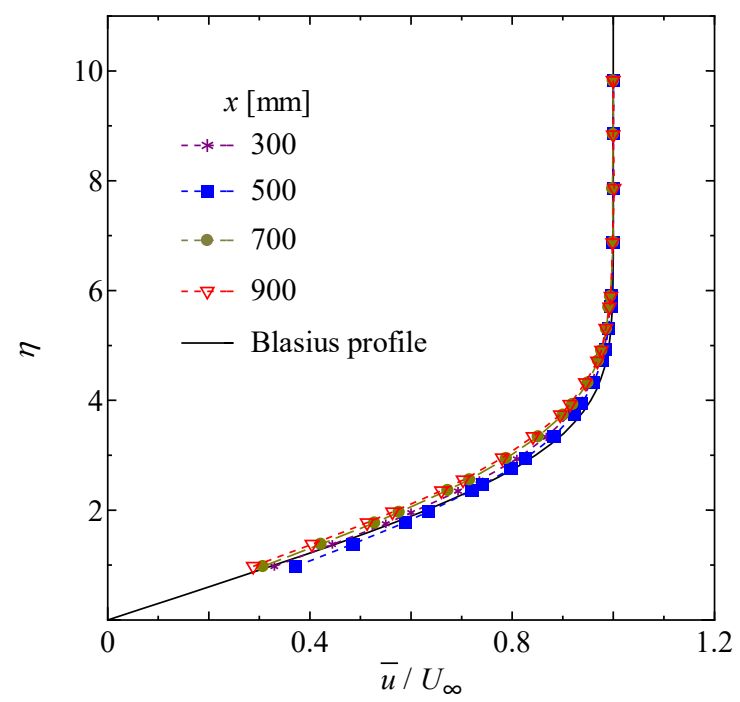

Fig. 2 Mean velocity profile for various streamwise locations at $z=0 \mathrm{~mm}$. The boundary layer is kept laminar in the measurement region.

intensity. Therefore, in this study, the deviations from the ensemble-averaged velocity signal were recognized herein as the fluctuating component of the velocity associated with turbulence, and their rms value was regarded as the turbulence intensity, which will be denoted as "the intensity of random components" hereafter. The definition of "the intensity of random components" is given as follows:

$$
\widetilde{u}(t)=\sqrt{\frac{1}{N} \sum_{i=1}^{N}\left(u_{i}(t)-<u(t)>\right)^{2}},
$$

where $N$ is the number of ensemble averaging which corresponds to the number of blowing/suction events; $u_{i}$ is the velocity of the $i$ th event; and $<u(t)>$ is the ensemble-averaged velocity.

\section{Results and discussion}

\subsection{Base flow and artificial disturbance}

In the absence of blowing and suction, the boundary layer was in a laminar state throughout the test section. Figure 2 shows the $y$-distribution of the time-averaged velocity $\bar{u}$ measured at various $x$ stations of $z=0 \mathrm{~mm}$. The vertical axis denotes the nondimensional height from the wall $(\eta)$ given as

$$
\eta=y \sqrt{\frac{U_{\infty}}{v x}}
$$

All of the velocity profiles agreed well with the Blasius profile throughout the measurement region, $x=300 \sim 1000 \mathrm{~mm}$. The same profiles were obtained at different spanwise locations, $z=-40$ and $40 \mathrm{~mm}$, at $x=1000 \mathrm{~mm}$. Thus, the base flow was laminar despite several holes in its surface at $x=300$ and $550 \mathrm{~mm}$.

Only the boundary-layer blowing/suction or the Triggering Jet was applied to the Blasius boundary layer. Figure 3(a) shows the contour map of the ensemble-averaged fluctuation $\left\langle u^{\prime}>\right.$ in the $z t$ plane at $\eta=2$ when only the Local Flow Destabilizer was activated. Figure 3(b) dipicts the same map when only the Triggering Jet was activated. The measurement locations were $100 \mathrm{~mm}$ downstream of each device $(x=500$ and $650 \mathrm{~mm})$. The time series data of $2^{19}$ obtained every $2 \mathrm{~mm}$ in the spanwise direction by the hotwire probe were converted into quasi-spatial data based on the Taylor's frozen-eddy hypothesis using the local time-averaged velocity of the base flow, $\bar{u}_{b}$. A total of $103 \sim 104$ runs were used to obtain each ensemble-averaged value. As shown in Fig. 3(a), a symmetric pattern of high- and low-speed regions appeared around the $z=0$ line when only the Local Flow Destabilizer was switched on. When only the Triggering Jet was turned on (Fig. 3(b)), a low-speed region at $z=0 \mathrm{~mm}$ and weak high-speed regions on both sides can be observed, although the pattern was narrower and much weaker than that of the Local Flow Destabilizer-only case. In both cases, the patterns gradually weakened further downstream, and no sign of turbulent transition could be observed throughout the measuring section. 
(a)

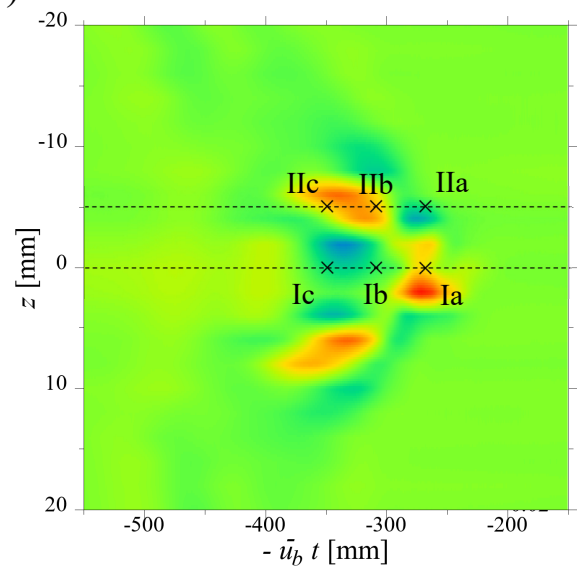

(b)

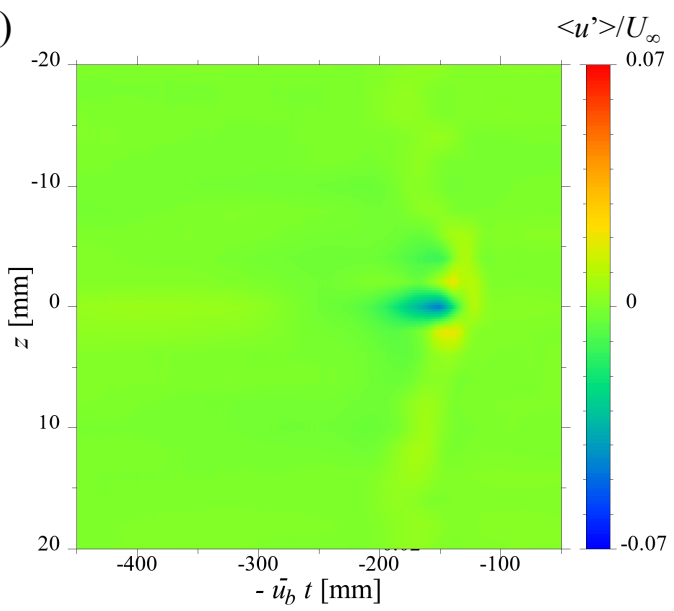

Fig. 3 Contour maps of the velocity fluctuation $\left\langle u^{\prime}>/ U_{\infty}\right.$ in the $\eta=2$ plane generated (a) only using the Local Flow Destabilizer at $x=300 \mathrm{~mm}$ and (b) only using Triggering Jet at $x=550 \mathrm{~mm}$. The measurements are performed at $x=550 \mathrm{~mm}$ for (a) and $x=650 \mathrm{~mm}$ for (b). The ejection timings of downstream Triggering Jet are marked by the cross symbols in (a).

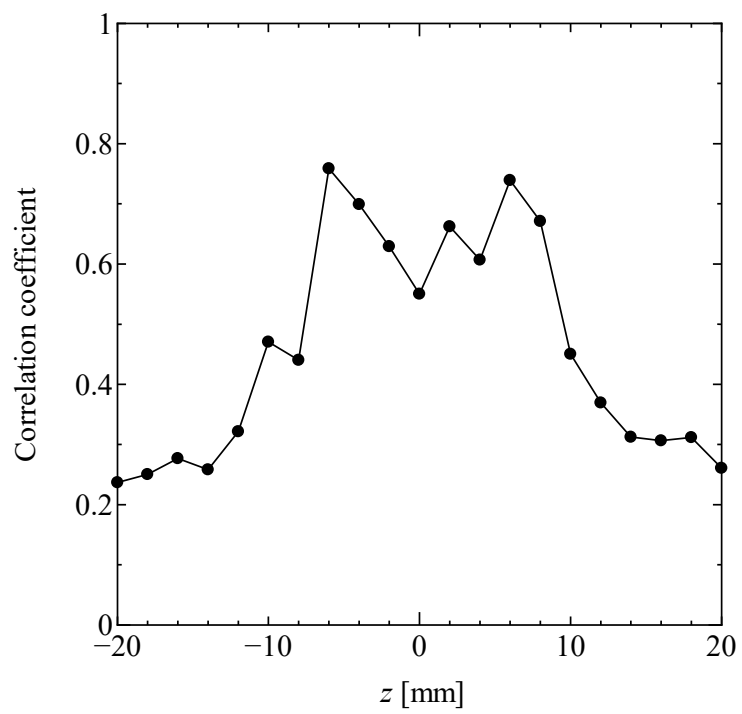

Fig. 4 Correlation coefficient between the ensemble-averaged velocity fluctuation $<u^{\prime}>$ and the instantaneous velocity fluctuation $u$ at $x=550 \mathrm{~mm}$ when the Local Flow Destabilizer is activated at arbitrary timings.

\subsection{Using the Local Flow Destabilizer and the Triggering Jet}

Next, both the Local Flow Destabilizer and the Triggering Jet were used together. The Local Flow Destabilizer was used to create a disturbed environment such that the ejection of the Triggering Jet downstream would become more effective. The Triggering Jet was ejected at the combination of three different timings and two spanwise locations, as shown by the cross symbols in Fig. 3(a). The three timings corresponded to ejecting the Triggering Jet at three streamwise locations marked as Ia, Ib, and Ic $\left(-\bar{u}_{b} t=-270,-310\right.$, and $-350 \mathrm{~mm}$, respectively). The cross symbols IIa, IIb, and IIc denote the result when the Triggering Jet location was shifted $5 \mathrm{~mm}$ in the negative $z$ direction. In the experiment, the Local Flow Destabilizer location was moved in the positive $z$ direction. It is to be noted here that, as shown in Fig, 4, the reproducibility of the disturbed field provided by the Local Flow Destabilizer is sufficiently good to apply the Taylor's frozen-eddy hypothesis. The correlation coefficient between the ensemble-averaged and instantaneous velocity fluctuations exhibits a high value of $0.45 \sim 0.75$ for the disturbed region of $\mathrm{z}=-10 \sim 10 \mathrm{~mm}$ at the ejection point of the Triggering Jet $\mathrm{x}=550 \mathrm{~mm}$.

Figure 5 shows the effect of the Triggering Jet ejected at three different timings, namely Ia, Ib, and Ic. The three figures in the left column represent timing Ia, those in the middle column represent timing Ib, and those in the right column represent timing Ic. At $x=650 \mathrm{~mm}$, which are the three figures on the top row of Fig. 5, the velocity fluctuation 


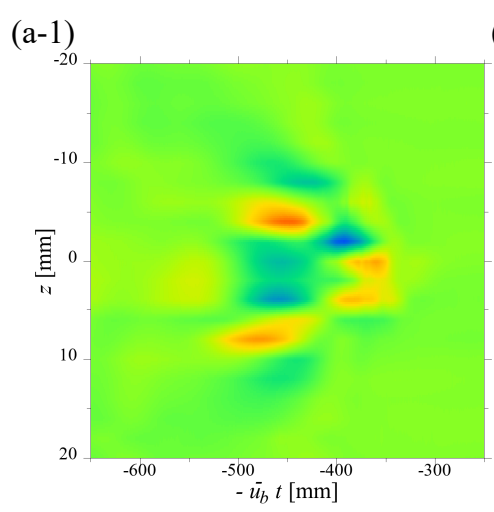

(b-1)

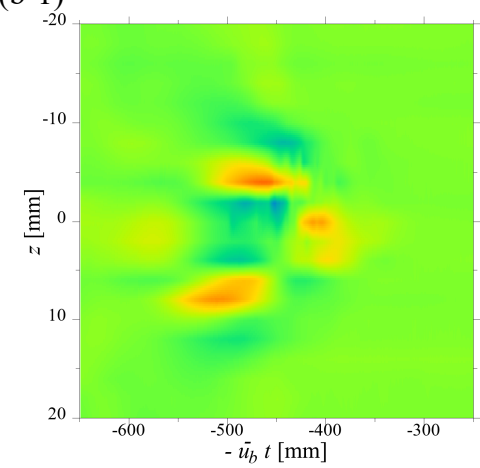

$(\mathrm{a}-2)$

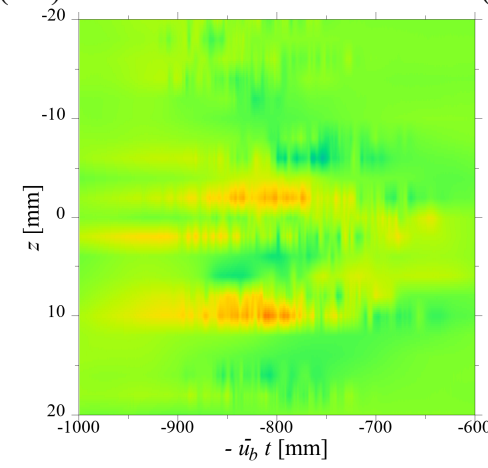

(b-2)

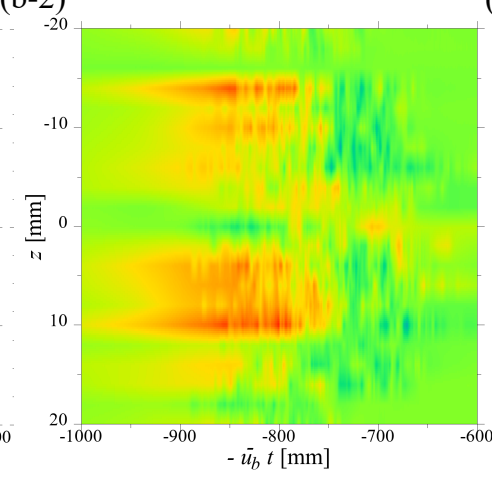

(b-3)

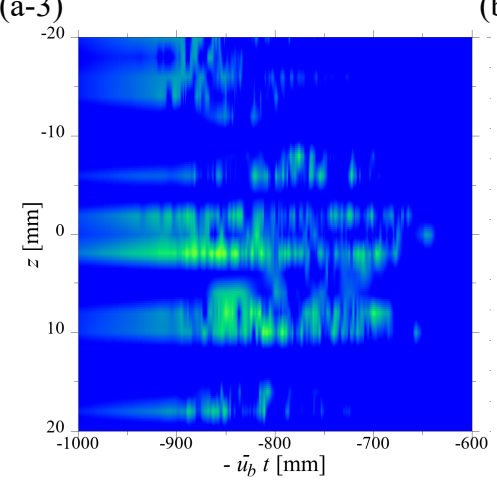

(c-1)

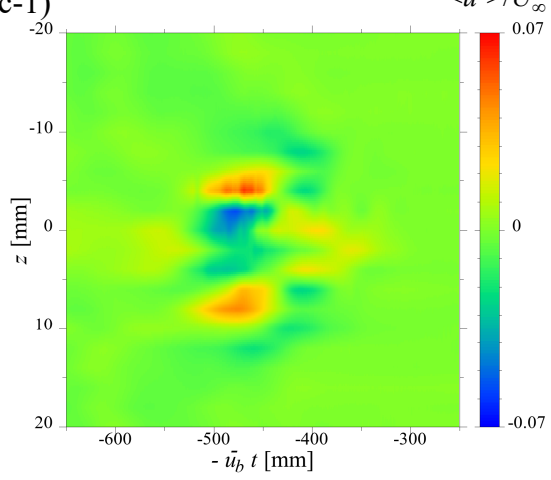

(c-2)
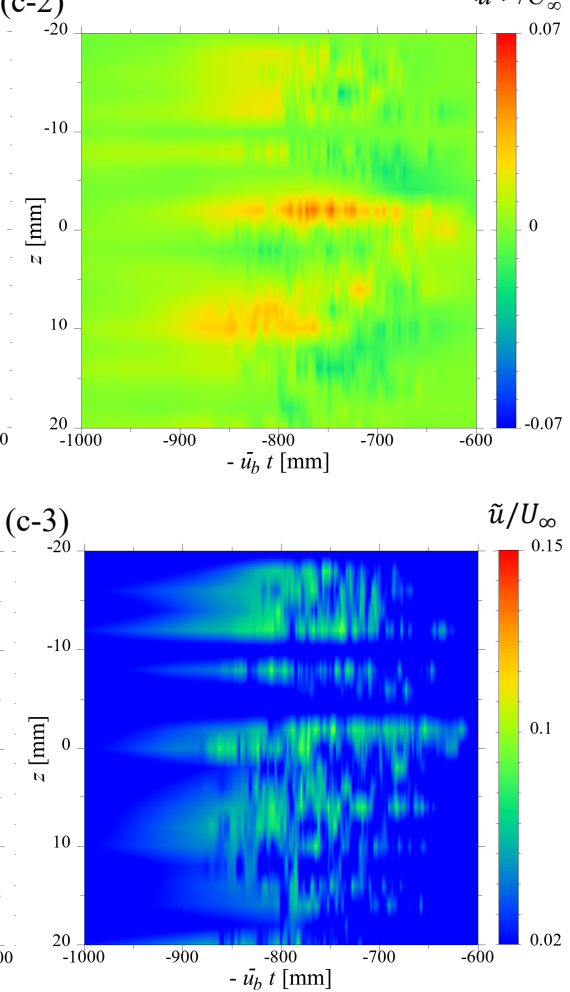

Fig. 5 Results of ejecting the Triggering Jet at different timings. The figures in the top row show the ensembleaveraged velocity fluctuation $\left\langle u^{\prime}>/ U_{\infty}\right.$ measured at $x=650 \mathrm{~mm}$ and $\eta=2.0$. The middle row figures show the same values while measured at $x=950 \mathrm{~mm}$. The bottom three figures depict the intensity of the random components $\widetilde{u} / U_{\infty}$ measured at $x=950 \mathrm{~mm}$ and $\eta=2.0$. The timing of jet ejection was Ia of Fig. 3 for the three figures on the left column, Ib for the middle column, and Ic for the figures at the right column. 

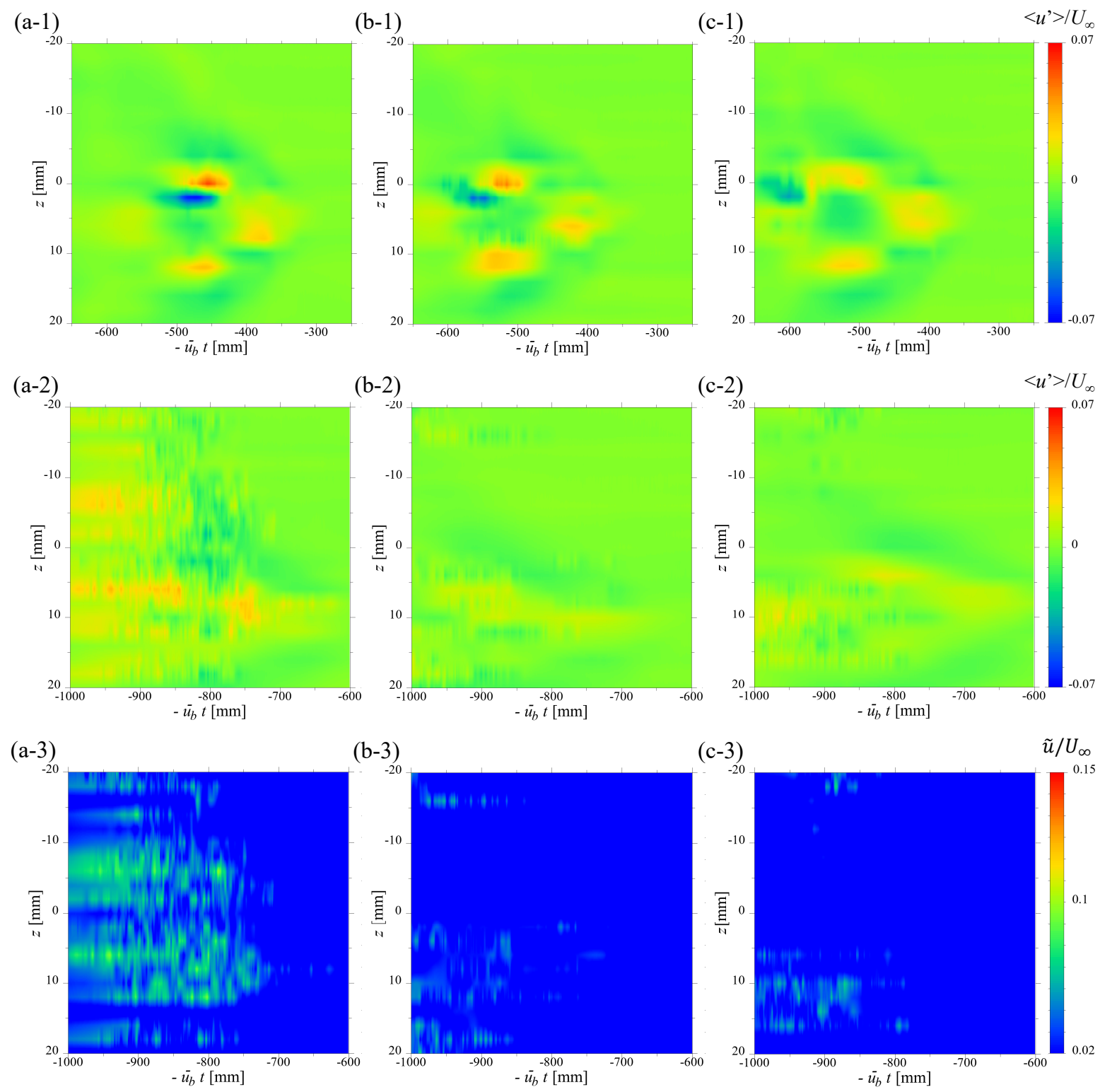

Fig. 6 Effect of the offset ejection of the Triggering Jet changing the timing. The figures on the top row show the ensemble-averaged velocity fluctuation $\left\langle u^{\prime}\right\rangle / U_{\infty}$ measured at $x=650 \mathrm{~mm}$ and $\eta=2.0$. The middle row figures show the same values while measured at $x=950 \mathrm{~mm}$. The bottom three figures depict the intensity of the random components $\widetilde{u} / U_{\infty}$ measured at $x=950 \mathrm{~mm}$ and $\eta=2.0$. The timing of jet ejection is at IIa for the left column, IIb for the middle column, and IIc for the right column. 


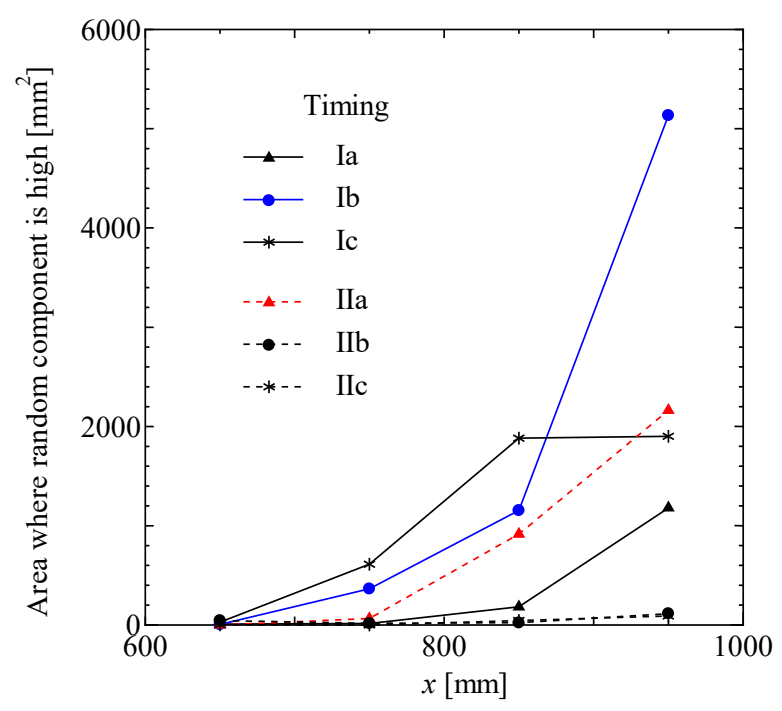

Fig. 7 Spreading of the high-random component area, $\widetilde{u} / U_{\infty} \geq 0.05$, in the $z t$ plane at $\eta=2$ for each case.

patterns were not so different from each other and from that introduced by the Local Flow Destabilizer alone (Fig. 3), irrespective of the jet ejection timings. At $x=950 \mathrm{~mm}$, shown in three figures in the middle row of Fig. 5, the differences caused by the jet ejection timing became obvious. Although the regions of high velocity fluctuations spread in both the streamwise and spanwise directions, the area and the intensity of the velocity fluctuations were much larger in the Ib case than in the others. The intensity of random components, which can be interpreted as an indicator of the local turbulence level, is presented in the bottom row of Fig. 5. The patterns were similar to the intensities of the velocity fluctuations measured at $x=950 \mathrm{~mm}$, indicating that the velocity fluctuations observed at $x=950 \mathrm{~mm}$ do not reflect the structures that appeared at the same timing. Therefore, the velocity fluctuations were not associated with the ejection of the Triggering Jet and were likely to be representing turbulence.

Figure 6 shows the results when the location of the Triggering Jet was shifted in the spanwise direction relative to the oncoming locally disturbed field. Three holes of the Local Flow Destabilizer at $x=300 \mathrm{~mm}$ and $z=0$ mm were moved by $5 \mathrm{~mm}$ in the positive spanwise direction. The Triggering Jet was ejected at timings IIa, IIb, and IIc, which are represented by the cross symbols in Fig. 3(a). Except for the relative spanwise locations of the Local Flow Destabilizer and the Triggering Jet, the figure is the same as Fig. 5. Figure 6 shows that, in the IIb and IIc cases, the velocity fluctuations created by the Triggering Jet decreased downstream, and the velocity fluctuations grew only when the Triggering Jet was ejected at timing IIa. Although the strength of the velocity fluctuations in the IIa case was not as strong as that in the Ib case, it was equivalent to that in the other cases in Fig. 5. Therefore, the optimum timing of the Triggering Jet ejection depended on its location to the oncoming locally disturbed region, which is interesting.

Figure 7 shows how the areas of a high random component $\widetilde{u} / U_{\infty} \geq 0.05$ in the $z t$ plane at $\eta=2$ spread. The measurements were made at the streamwise stations, $x=650,750,850$, and $950 \mathrm{~mm}$. The disturbed areas were estimated herein from the ensemble-averaged fields, which implied that the cases in which the introduced fluctuations amplified or damped downstream were all included in the figure. The increase of the disturbed area between $x=850$ and $950 \mathrm{~mm}$ was remarkable for timing Ib. The comparisons of the ensemble-averaged velocity $<u>$ are shown in Fig. 8(a) for five different instants, including jet ejection timings Ia, Ib, and Ic. The color map of the ensemble-averaged velocity fluctuation $<u^{\prime}>$ at $z=0 \mathrm{~mm}$ is also shown in Fig. 8(b), where the dotted lines represent the timings, Ia, Ib and Ic. The $<u>$ profiles, in Fig. 8 (a), were close to the Blasius profile, regardless of the work by the Local Flow Destabilizer upstream. In Fig. 8 (b), the influence of activating the Local Flow Destabilizer appears as an inclined high-velocity region followed by a low-velocity region. The most favorable timing $\mathrm{Ib}\left(-\bar{u}_{b} t=-310 \mathrm{~mm}\right)$ appeared to be ejecting the Triggering Jet between the high- and low-speed regions.

The necessary strength of the Triggering Jet for the generation of a localized turbulent region was evaluated herein using factor $\gamma$, which represents the probability of turbulence generation. Factor $\gamma$ is defined as follows:

$$
\gamma=\frac{\text { Number of generated turbulent regions }}{\text { Number of trials }}
$$

where the number of trials represents the number of the jet ejections, which was 418 in total. The generation of local 

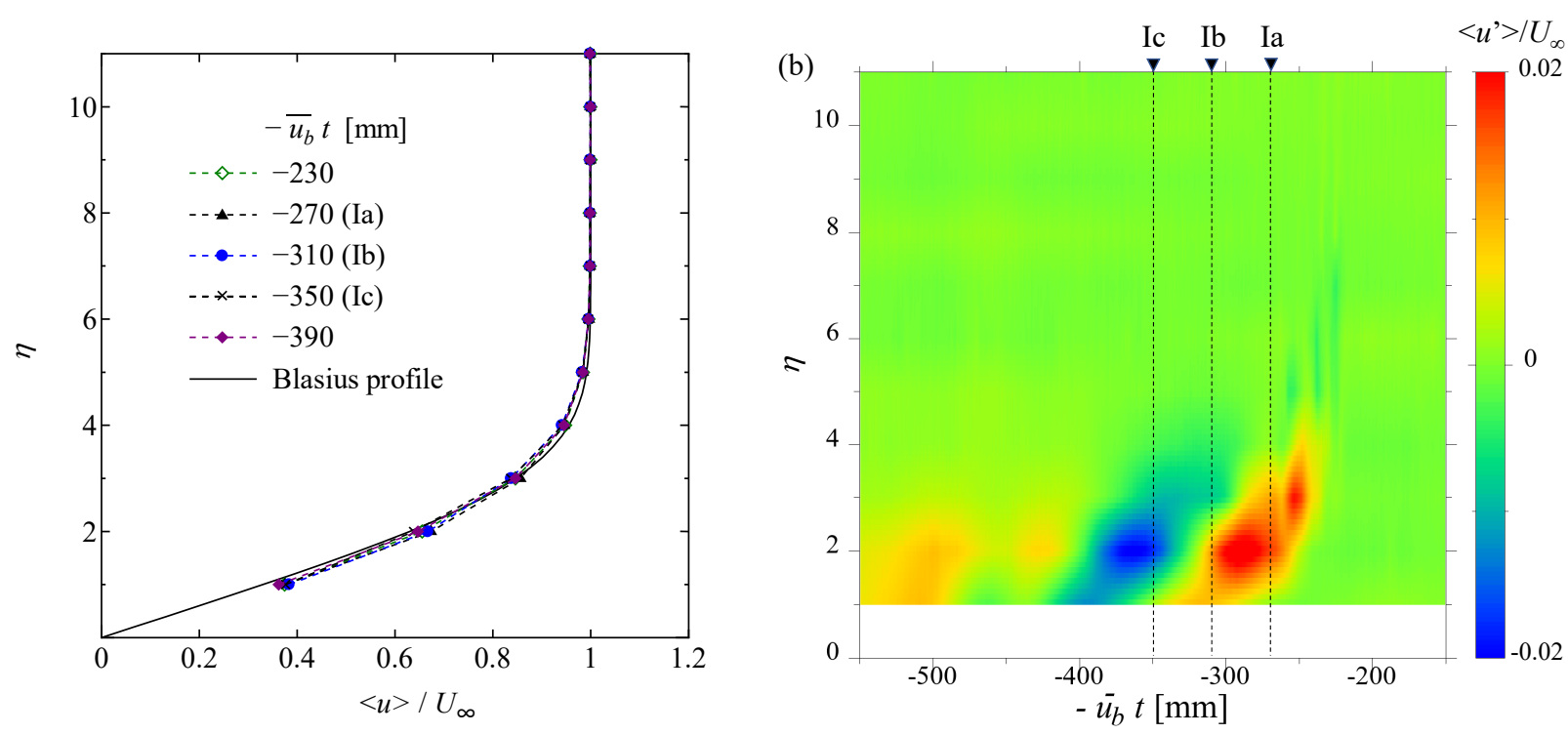

Fig. 8 Comparison of (a) the ensemble-averaged velocity $\langle u(t)>$ profiles and (b) the distribution of fluctuation $\left.<u^{\prime}\right\rangle / U_{\infty}$ at $x=550 \mathrm{~mm}$ and $z=0 \mathrm{~mm}$. The dotted lines in (b) represent the timings $-\bar{u}_{b} t=-270$ (Ia), -310 (Ib), and $-350 \mathrm{~mm}(\mathrm{Ic})$ in the $\eta t$ plane.

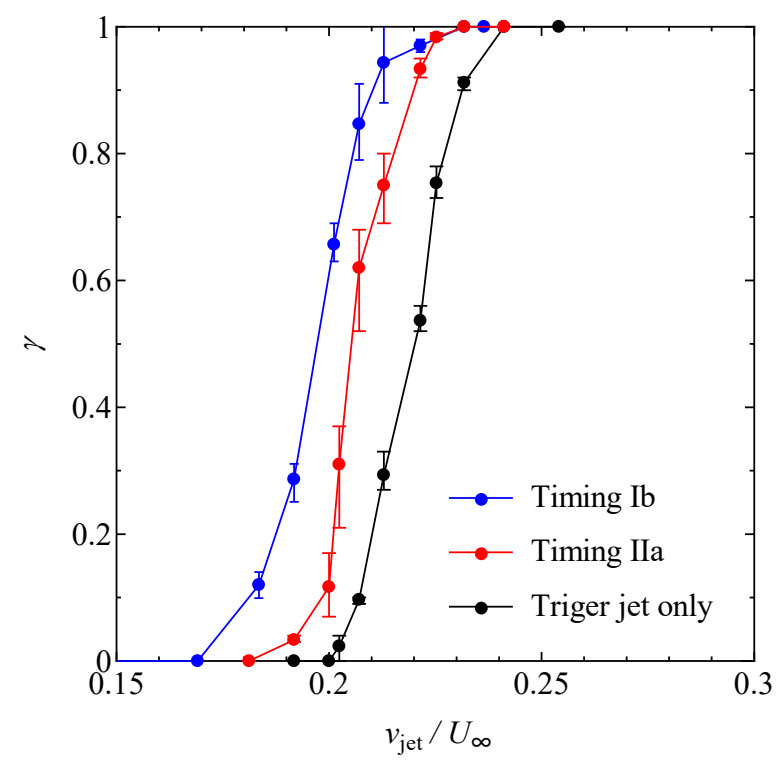

Fig. 9 Comparison of probability $\gamma$ for the turbulent regions to be generated. Cases Ib and IIa and case without the Local Flow Destabilizer are compared at $x=950 \mathrm{~mm}, \eta=2$, and $z=0 \mathrm{~mm}$. 
(a)

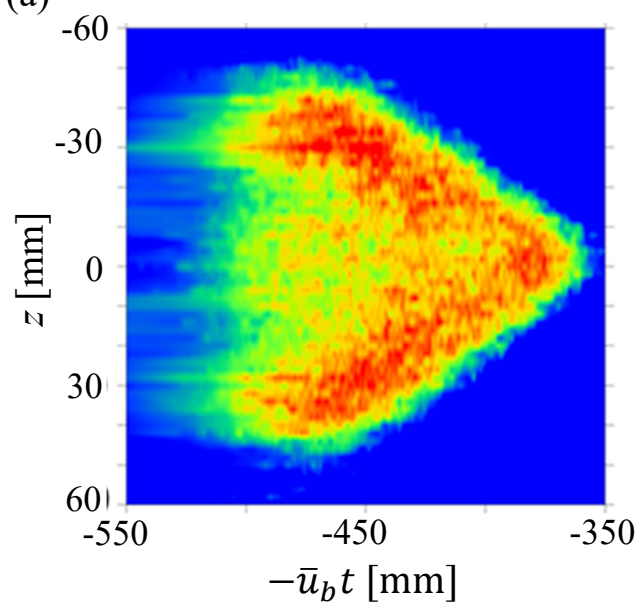

(b)

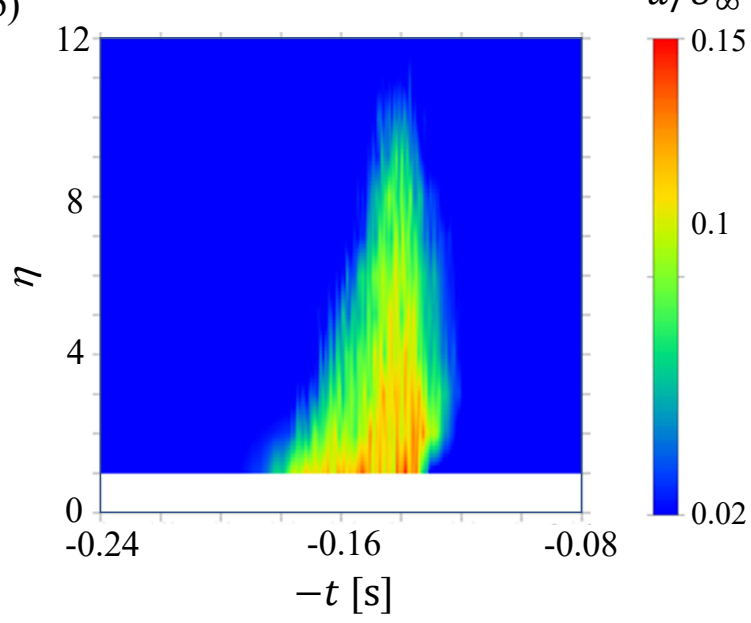

Fig. 10 Triggering Jet operated at timing $\mathrm{Ib}$ with an increased velocity of $v_{\text {jet } / \mathrm{U}_{\infty}}=0.27$. The contour maps represent the intensity of the random components $\widetilde{u} / U_{\infty}$ at $x=950 \mathrm{~mm}$ in (a) the $z t$ plane at $\eta=1$ and (b) the $\eta t$ plane at $z=0 \mathrm{~mm}$.

turbulent region was judged based on whether or not the amplitude of the velocity fluctuations exceeded $10 \%$ of the freestream velocity. Each experiment was conducted thrice under the same conditions. Their average value was then calculated.

Figure 9 shows the comparison of the probability $\gamma$ of the turbulent region to be generated for the cases with the most effective timings Ib and IIa for each of the two configurations with different spanwise offsets. The case when the Triggering Jet was ejected into an undisturbed Blasius boundary layer is also plotted in the figure for reference. The red, blue, and black symbols indicate the results for timings Ib and IIa, and the "Triggering Jet only" case. The maximum and minimum values among the three measurements are shown by the error bar. The probability of generating a turbulent region increased with the jet velocity. For the two cases (i.e., Ib and IIa), the jet velocities necessary to generate turbulent regions were lower compared to those in the "Triggering Jet only" case. The figure clearly shows that creating a destabilized environment upstream and ejecting the Triggering Jet at the right timing can reduce the strength of the Triggering Jet necessary to generate a turbulent region.

\subsection{Investigation of the local turbulent region using a stronger jet}

Although Figs. 5 and 6 show the spread of the turbulent region, their shapes were ambiguous because the results were the ensemble averages of over 100 experimental runs. The size, shape, and location of the turbulent region were different in every run. Therefore, the strength of the jet ejection $v_{\text {jet }}$ of the Triggering Jet was increased to $27 \%$ of the freestream velocity to clearly capture the feature of the turbulent region. Three cases, namely Ib, IIa, and "Triggering Jet only" were tested. Figure 10 shows the contour maps of the intensity of random components at $x=950 \mathrm{~mm}$ in the $z t$ plane at $\eta=1$ and in the $\eta t$ plane at $z=0 \mathrm{~mm}$ for case $\mathrm{Ib}$. An arrow-shaped pattern was clearly observed in the $z t$ plane parallel to the wall. The leading edge on the downstream side overhung and extended beyond the outer edge of the boundary layer, $\eta=5$. By contrast, the tailing edge on the upstream side gradually narrowed toward the wall. The same patterns were obtained for the IIa and "Triggering Jet only" cases. These geometric characteristics were in a good agreement with those of a typical turbulent spot, which often appears in the transitional boundary layer (Henningson et al. 1987; Riley and Gad-el-Hak 1985; Gutmark et al. 1987).

The intensity of random components can be used to identify a turbulent region. In this study, a turbulent state is defined as the state when the following condition is satisfied:

$$
\frac{\widetilde{u}}{U_{\infty}} \geq 0.1 \text {. }
$$

Figure 11 represents the locations of the spot's leading and trailing edges. Here, the horizontal and vertical axes represent the elapsed time and the distance from the virtual origin of the spots, respectively. The virtual origin is defined as the point where the line connecting the spot's leading edge locations and the line connecting the spot's trailing edge locations intersect, when measured in the $z t$ plane of $\eta=1$. Although the turbulent region initially appeared to be disordered, 


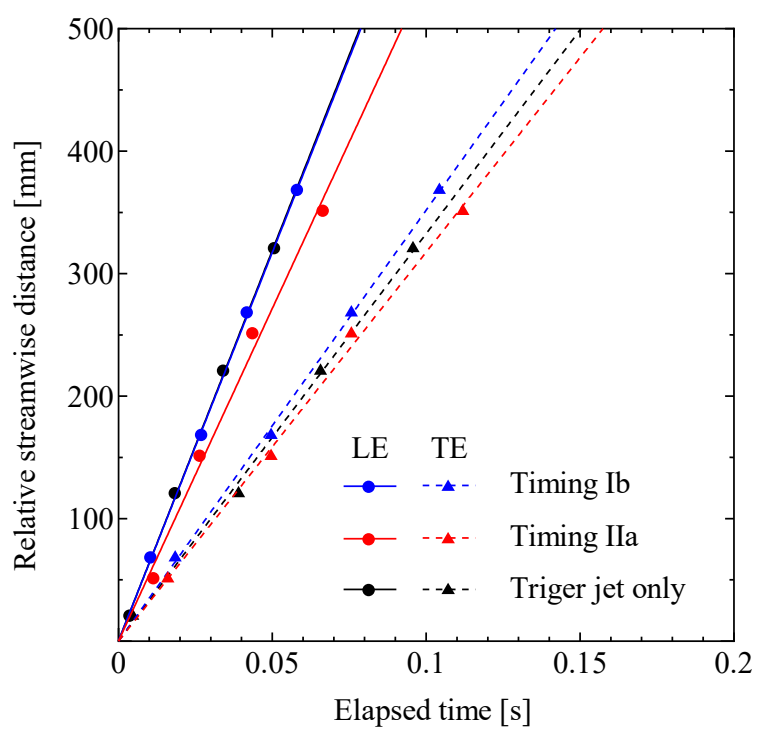

Fig. 11 Locations of the leading and trailing edges of the turbulent spot. Measured in the $z t$ plane of $\eta=1$.

it gradually developed into an arrow-shaped pattern. The streamwise length of the spot increased in proportion to the elapsed time in all cases. In other words, the destabilized flow field prepared upstream using the Local Flow Destabilizer had little influence on the growth of turbulent spots, although it influenced the generation of spots.

\section{Conclusions}

A wind tunnel experiment was performed to investigate the suitable conditions in which a localized turbulent region can be easily generated in a laminar boundary layer. An unstable region was prepared upstream using the Local Flow Destabilizer, which was a combination of a short-duration jet and suctions. Another jet was then ejected downstream by the Triggering Jet device, changing the timing and the spanwise location with respect to the passage of the convected unstable region. Although neither the Local Flow Destabilizer nor the Triggering Jet could generate turbulence by itself, the combination of the two generated a localized turbulent region, that is, a turbulent spot. Ejecting the Triggering Jet to an area between a high-speed region and a low-speed region generated by the Local Flow Destabilizer was effective.

\section{References}

Andersson, P., Brandt, L., Bottaro, A. and Henningson, D. S., On the breakdown of boundary layer streaks, Journal of Fluids Mechanics, Vol. 428 (2001), pp. 29-60.

Asai, M., Minagawa, M. and Nishioka, M., The instability and breakdown of a near-wall low-speed streak, Journal of Fluids Mechanics, Vol. 455 (2002), pp. 289-314.

Asai, M., Konishi, Y., Oizumi, Y. and Nishioka, M., Growth and breakdown of low-speed streaks leading to wall turbulence, Journal of Fluid Mechanics, Vol. 586 (2007), pp. 371-396.

Eloffson, P. A, Kawakami, M. and Alfredsson, P. H, Experimental investigation on the stability of streamwise streaks in plane Poiseuille flow, Phisics of Fluids., Vol. 11 (1999), pp. 915-930.

Emmons, H. W., The Laminar-Turbulent Transition in a Boundary Layer, Journal of the Aeronautical Sciences., Vol. 18, No. 7 (1951), pp. 490-498.

Fransson, J. H. M., Turbulent spot evolution in spatially invariant boundary layers, Physical Review E., Vol. 81, 035301 (2010), pp. 1-4.

Gutmark, E. and Blackwelder, R. F., On the structure of a turbulent spot in a heated laminar boundary layer, Experimental in Fluids, Vol. 5 (1987), pp. 217-229.

Henningson, D. S., Spalart, P. and Kim, J., Numerical simulations of turbulent spots in plane Poiseuille and boundary-layer flow, Physics of Fluids, Vol. 30 (1987), pp. 2914-2917.

Herbert, T., Secondary instability of boundary layers, Annual Review of Fluid Mechanics, Vol. 20 (1988), pp. 487-526. 
Riley J.J. and Gad-el-Hak M.,The dynamics of turbulent spots. In: Davis S.H., Lumley J.L. (eds) Frontiers in Fluid Mechanics (1985). Springer.

Schlatter, P., Brandt, L., de Lange, H. C. and Henningson, D. S., On streak breakdown in bypass transition, Physics of Fluids, Vol. 20 (2008), 101505.

Schubauer, G. B. and Klebanoff, P. S., Contributions on the mechanics of boundary-layer transition, NACA Technical Report, 1289 (1955).

Levin, O. and Henningson, D. S., Turbulent spots in the asymptotic suction boundary layer, Journal of Fluid Mechanics., vol. 584 (2007), pp. 397-413.

Makita, H. and Nishizawa, A., Characteristics of internal vortical structures in a merged turbulent spot, Journal of Turbulence., Vol. 2 (2001), pp. 1-14.

Narashima, R., The laminar-turbulent transition zone in the boundary layer, Progress in Aerospace Sciences, Vol. 22 (1985), pp. 29-80.

Perry, A., Lim, T. T. and Teh, E. W., A visual study of turbulent spots, Journal of Fluid Mechanics, Vol. 104 (1981), pp. 387-405.

Sankaran, R., Antonia, R. A., Bisset, D. K and Sokolov, M., Flow patterns and organzation within a turbulent spot, Physics of Fluids. A: Fluid Dynamics 3 (1991), pp. 1560-1571.

Seifert, A., Zilberman, M. and Wygnanski, I., On the simultaneous measurements of two velocity components in the turbulent spot, Journal of Engineering Mechanics., Vol.28 (1994), pp. 43-54.

Uehara, M., Shigeta, M., Izawa, S. and Fukunishi, Y., Generation of turbulent spots in an environment with streaky structure, The Proceedings of Autumn Conference of Tohoku Branch, Vol. 2010.46 (2010), pp. 57-58. (in Japanese)

Yoshikawa, J., Nishio, Y., Izawa, S. and Fukunishi, Y., Key structure which initiate laminar-turbulent transition process in boundary layer with streaky structures, Transactions of the JSME, Vol. 84, No. 857 (2018a), 17-00478. (in Japanese)

Zhong, S., Kittichaikan, C., Hadson, H. P. and Ireland, P. T., Visualisation of turbulent spots under the influence of adverse pressure gradient, Experiments of Fluids., Vol. 28 (2000), pp. 385-393. 